\title{
A REMARK ON ZAK'S THEOREM ON TANGENCIES
}

\author{
JOSÉ CARLOS SIERRA
}

\begin{abstract}
We present a slightly different formulation of Zak's theorem on tangencies as well as some applications. In particular, we obtain a better bound on the dimension of the dual variety of a manifold and we classify extremal and next-to-extremal cases when its secant variety does not fill up the ambient projective space.
\end{abstract}

\section{Introduction}

In this note, we state Zak's theorem on tangencies ([15, Theorem 0], see also [16, Ch. I, Corollary 1.8]) for non-singular complex varieties in the following way:

Theorem 1.1 (Reformulation of Zak's theorem on tangencies). Let $X \subset \mathbb{P}^{N}$ be a non-degenerate manifold of dimension $n$, and let $L \subset \mathbb{P}^{N}$ be a linear subspace of dimension $m$ which is tangent to $X$ along a closed subvariety $Y \subset X$ of dimension $r$. Then

$$
r \leq \min \{m-n, \operatorname{dim} S X-1-n\} .
$$

The former statement appears to have some advantages. The new inequality $r \leq$ $\operatorname{dim} S X-1-n$ is vacuous if the secant variety of $X$ (denoted by $S X$ ) fills up $\mathbb{P}^{N}$, but it is significant when $S X \neq \mathbb{P}^{N}$. For instance, this is always the case if $m=N-1$. In this setting, Zak's theorem on tangencies has several consequences (see [10] for an account) that can be sharpened, thanks to Theorem 1.1. Let $s:=\operatorname{dim} S X$ and $c:=N-s$.

Corollary 1.1. Let $X \subset \mathbb{P}^{N}$ be a non-degenerate manifold of dimension $n$, and let $X^{*} \subset \mathbb{P}^{N *}$ denote its dual variety (of dimension $n^{*}$ ). The following holds:

(i) The twisted normal bundle $N_{X / \mathbb{P}^{N}}(-1)$ is $k$-ample for $k \geq s-1-n$ (cf. [10, Example 6.3.7]).

(ii) $n^{*} \geq n+c\left(c f .\left[10\right.\right.$, Corollary 3.4.20]). In particular, if $S X \neq \mathbb{P}^{N}$ then $X^{*}$ is a singular variety.

(iii) If $s \leq 2 n-1$ (resp. $2 n-2)$ then every hyperplane section of $X$ is reduced (resp. normal) (cf. [10, Corollary 3.4.19]).

Some examples of manifolds satisfying the equality $n^{*}=n$ (and hence $S X=\mathbb{P}^{N}$ ) are given by hypersurfaces in $\mathbb{P}^{n+1}$, Segre embeddings $\mathbb{P}^{1} \times \mathbb{P}^{n-1} \subset \mathbb{P}^{2 n-1}$, the Grassmannian $\mathbb{G}(1,4) \subset \mathbb{P}^{9}$ and the 10 -dimensional spinor variety $S_{4} \subset \mathbb{P}^{15}$. Moreover, these are the only examples under the additional assumption $3 n \leq 2 N$ by Ein $[2$, Theorem 4.5] (cf. Remark 2.3). On the other hand, if $S X \neq \mathbb{P}^{N}$ we show that furthermore $n^{*} \geq n+c+1$ and manifolds satisfying the equality are classified, giving a new characterization of the Veronese surface:

Received by the editors January 27, 2011. 
Theorem 1.2. Let $X \subset \mathbb{P}^{N}$ be a non-degenerate manifold of dimension $n$. If $S X \neq$ $\mathbb{P}^{N}$ then $n^{*} \geq n+c+1$, with equality if and only if $X$ is either a curve or the Veronese surface in $\mathbb{P}^{\frac{1}{5}}$.

Going one step further, let us consider the next-to-extremal case when $S X \neq \mathbb{P}^{N}$. If $n \leq 3$ it is easy to see (cf. Remark 2.2) that $n^{*}=n+c+2$ if and only if $X$ is either a surface, or a dual defective threefold (i.e., a scroll over a curve), or a secant defective threefold (see [3] for the classification). On the other hand, for $n \geq 4$ we get the following:

Theorem 1.3. Let $X \subset \mathbb{P}^{N}$ be a non-degenerate manifold of dimension $n \geq 4$. If $S X \neq \mathbb{P}^{N}$ and $n^{*}=n+c+2$, then $X$ is a scroll over a manifold $W$ and $\operatorname{dim} W \leq 2$.

If $\operatorname{dim} W=2$ we will prove in Theorem 3.1 that the Segre embedding of $\mathbb{P}^{2} \times \mathbb{P}^{n-2}$ is the only scroll as in Theorem 1.3, so we actually get the following refinement:

Theorem 1.4. Let $X \subset \mathbb{P}^{N}$ be a non-degenerate manifold of dimension $n \geq 4$ such that $S X \neq \mathbb{P}^{N}$. Then $n^{*}=n+c+2$ if and only if $X$ is either a scroll over a curve, or (an isomorphic projection of) the Segre embedding $\mathbb{P}^{2} \times \mathbb{P}^{n-2} \subset \mathbb{P}^{3 n-4}$.

\section{Proofs}

Theorem 1.1 is a consequence of the following application of the Fulton-Hansen connectedness theorem [5]. First, we recall the definition of the relative tangent (resp. secant) variety. Given a subvariety $Y \subset X$, we define $T(Y, X):=\cup_{y \in Y} T_{y} X$, where $T_{y} X \subset \mathbb{P}^{N}$ denotes the embedded tangent space to $X$ at $y \in Y$, and $S(Y, X) \subset \mathbb{P}^{N}$ as the closure of $\left\{z \in \mathbb{P}^{N} \mid \exists y \in Y, \exists x \in X\right.$ with $\left.z \in\langle y, x\rangle\right\}$.

Theorem 2.1. Let $X \subset \mathbb{P}^{N}$ be a non-degenerate manifold of dimension $n$, and let $Y \subset X$ be a closed subvariety of dimension $r$. Then either $\operatorname{dim} T(Y, X)=r+n$ and $\operatorname{dim} S(Y, X)=r+n+1$, or else $T(Y, X)=S(Y, X)$.

Proof. See [16, Ch. I, Theorem 1.4].

We can now prove our results:

Proof of Theorem 1.1. Let $L \subset \mathbb{P}^{N}$ be a linear subspace of dimension $m$ which is tangent to $X$ along $Y$. Then $T(Y, X) \subset L$, but $S(Y, X) \not \subset L$ as $X \subset \mathbb{P}^{N}$ is nondegenerate. Therefore $T(Y, X) \neq S(Y, X)$, and hence $r+n=\operatorname{dim} T(Y, X) \leq \operatorname{dim} L=$ $m$ by Theorem 2.1. But Theorem 2.1 also yields $r+n+1=\operatorname{dim} S(Y, X) \leq \operatorname{dim} S X$, so $r \leq \min \{m-n, \operatorname{dim} S X-1-n\}$.

Remark 2.1. If $S X \neq \mathbb{P}^{N}$ and $m=N-1$, the case we are more interested in, the new bound $r \leq s-1-n$ is sharp. For example, equality holds for Severi varieties [16, Ch. IV] when $Y \subset X$ is an $n / 2$-dimensional quadric.

Proof of Corollary 1.1. According to Theorem 1.1, the dimension of the fibres of the second projection of the conormal variety $\mathcal{P}_{X}:=\left\{(x, H) \mid T_{x} X \subset H\right\} \subset X \times \mathbb{P}^{N^{*}}$ is bounded by $s-1-n$. So $N_{X / \mathbb{P}^{N}}(-1)$ is $k$-ample for $k \geq s-1-n$. This proves (i). Since $\operatorname{dim} \mathcal{P}_{X}=N-1$, we get $n^{*} \geq n+c$. Assume now that $X^{*}$ is smooth. Then $\operatorname{dim}\left(X^{*}\right)^{*} \geq n^{*} \geq n+c$, and Segre's reflexivity theorem $\left(X^{*}\right)^{*}=X([13]$, see also [9] for a detailed account) yields $c=0$, whence $S X=\mathbb{P}^{N}$ proving (ii). Part (iii) is an immediate consequence of Theorem 1.1. 
The main ingredients of the proof of Theorem 1.2 are Zak's classification of Severi varieties and Ein's bound on the defect of subcanonical manifolds [2, Theorem 4.4]:

Proof of Theorem 1.2. Assume $n \geq 2$ and $n^{*} \leq n+c+1$. Let $\operatorname{def}(X)$ and $\delta(X)$ denote the dual and secant defect of $X \subset \mathbb{P}^{N}$, respectively. As $\operatorname{def}(X):=N-1-n^{*}$ and $\delta(X):=2 n+1-s$, the inequality $n^{*} \leq n+c+1$ is equivalent to the inequality $\operatorname{def}(X)+\delta(X) \geq n-1$. Since we assume $S X \neq \mathbb{P}^{N}$, we get $\delta(X) \leq n / 2$ by Zak's theorem on linear normality [16, Ch. II, Corollary 2.11] and equality holds if and only if $X \subset \mathbb{P}^{N}$ is a Severi variety. Assume first that the Picard group of $X$ is cyclic. Then $\operatorname{def}(X) \leq(n-2) / 2$ by Ein [2, Theorem 4.4], and hence

$$
n-1 \leq \operatorname{def}(X)+\delta(X) \leq \frac{n-2}{2}+\frac{n}{2}=n-1
$$

implies that $X \subset \mathbb{P}^{N}$ is a Severi variety with $\operatorname{def}(X)=(n-2) / 2$. So $X$ is the Veronese surface in $\mathbb{P}^{5}$. Assume now that the Picard group of $X$ is not cyclic. Then $\delta(X) \leq 2$ by the Barth-Larsen theorem, as otherwise $X \subset \mathbb{P}^{N}$ could be isomorphically projected into $\mathbb{P}^{2 n-2}$ (see for instance [10, Corollary 3.2.3]). Therefore $\operatorname{def}(X) \geq n-3$, whence $\operatorname{def}(X)=n-2$ by Landman's parity theorem (unpublished, see [2, Theorem 2.4]) and $X \subset \mathbb{P}^{N}$ is a scroll over a curve by Ein [2, Theorem 3.2]. This yields $1 \leq \delta(X) \leq 2$, contradicting Lemma 3.2.

Remark 2.2. (i) The bound $n^{*} \geq n+c$ given in Theorem 1.1 is equivalent to the bound $\operatorname{def}(X)+\delta(X) \leq n$. Furthermore, if $S X \neq \mathbb{P}^{N}$ then $\operatorname{def}(X)+\delta(X) \leq n-1$ by Theorem 1.2. To the best of the author's knowledge, these relations involving both dual and secant defects appear to be new.

(ii) For $n \geq 3$ the bound obtained in Theorem 1.2 is equivalent to the bound $\operatorname{def}(X)+\delta(X) \leq n-2$. This can be seen as a refinement of the inequality $\operatorname{def}(X) \leq$ $n-2$ of Landman and Zak when $S X \neq \mathbb{P}^{N}$ (cf. [16, Ch. I, Remark 2.7]).

(iii) Besides curves and the Veronese surface, the bound $n^{*} \geq n+c+2$ (or equivalently $\operatorname{def}(X)+\delta(X) \leq n-2)$ is sharp. Equality holds for surfaces with $\delta(X)=0$, threefolds with $\delta(X)=\overline{1}$, scrolls over curves with $\delta(X)=0$ and the Segre embeddings $\mathbb{P}^{2} \times \mathbb{P}^{n-2} \subset \mathbb{P}^{3 n-4}$ with $n \geq 4$. We will prove in the sequel that these are actually the only ones.

The key of the proof of Theorem 1.3 is a recent characterization of scrolls among dual defective manifolds obtained by Ionescu and Russo in [6]:

Proof of Theorem 1.3. Let $n^{*}=n+c+2$, that is, $\operatorname{def}(X)+\delta(X)=n-2$. If $X$ is a scroll over a manifold $W$ then $\delta(X) \leq 2$ by the Barth-Larsen theorem. Thus $n-4 \leq \operatorname{def}(X)=n-2 \operatorname{dim} W$, so $\operatorname{dim} W \leq 2$. If $X$ is not a scroll, we can assume $\operatorname{def}(X) \leq(n+1) / 3$ by Ionescu and Russo [6, Corollary 3.7] and $\delta(X) \leq(n-1) / 2$ by the classification of Severi varieties. Therefore,

$$
n-2=\operatorname{def}(X)+\delta(X) \leq \frac{n+1}{3}+\frac{n-1}{2}
$$

yields $n \leq 11$ and, in view of Landman's parity theorem, we get $(n, \operatorname{def}(X), \delta(X)) \in$ $\{(5,1,2),(6,2,2),(9,3,4)\}$. The first two cases are excluded by Ein $[1$, Theorems 5.1 and 5.2]. In the third case, $X$ is a Fano manifold of dimension 9 with cyclic Picard group generated by the hyperplane section and index $(n+\operatorname{def}(X)+2) / 2=7$ (see $[2$, 
Lemma 4.2]), so it is ruled out by Mukai's classification of Fano manifolds of coindex 3 (see [11]).

Remark 2.3. (i) In a similar way, we can prove that a non-degenerate $n$-fold $X \subset \mathbb{P}^{N}$ with $n^{*}=n$ is either the Segre embedding $\mathbb{P}^{1} \times \mathbb{P}^{n-1} \subset \mathbb{P}^{2 n-1}$, or else $4 n+5 \geq 3 N$ and equality holds if and only if $X$ is the 10-dimensional spinor variety $S_{4} \subset \mathbb{P}^{15}$. Since $n^{*}=n$ we deduce $S X=\mathbb{P}^{N}$ by Corollary 1.1 , and hence $\delta(X)=2 n+1-N$. We point out that $n^{*}=n$ is equivalent to $\operatorname{def}(X)+\delta(X)=n$. If $X$ is a scroll then $\delta(X) \leq 2$, whence $\operatorname{def}(X) \geq n-2$. Therefore $\operatorname{def}(X)=n-2, \delta(X)=2$ and $X$ is the Segre embedding $\mathbb{P}^{1} \times \mathbb{P}^{n-1} \subset \mathbb{P}^{2 n-1}$ by Proposition 3.1. On the other hand, if $X$ is not a scroll then $\operatorname{def}(X) \leq(n+2) / 3$ and equality holds if and only if $X$ is the 10-dimensional spinor variety by Ionescu and Russo [6, Corollary 3.7]. Thus $\delta(X) \geq(2 n-2) / 3$, and hence $4 n+5 \geq 3 N$, with equality if and only if $X$ is the 10-dimensional spinor variety $S_{4} \subset \mathbb{P}^{15}$.

(ii) If $n^{*}=n$ and one furthermore assumes that $3 n \leq 2 N$ (cf. [2, Theorem 4.5]) then one also gets hypersurfaces and the Grassmannian $\mathbb{G}(1,4) \subset \mathbb{P}^{9}$, as in $[6$, Corollary 3.9].

Proof of Theorem 1.4. In Theorem 1.3, if $\operatorname{dim} W=2$ then $\operatorname{def}(X)=n-4$ and hence $\delta(X)=2$, since $\operatorname{def}(X)+\delta(X)=n-2$. So we conclude in view of Theorem 3.1.

\section{A result on secant defective scrolls over surfaces}

In this section we prove the results on scrolls quoted in Section 2. We say that $X_{W} \subset \mathbb{P}^{N}$ (or simply $X$ ) is a scroll if there exists a vector bundle $\mathcal{E}$ over a manifold $W$ such that $X_{W} \cong \mathbb{P}_{W}(\mathcal{E})$ and the fibres of the map $\pi: X_{W} \rightarrow W$, that we denote by $F_{w}$ for $w \in W$, are linearly embedded in $\mathbb{P}^{N}$. An equivalent definition of scroll is the following. Let $\mathbb{G}(k, N)$ denote the Grassmannian of $k$-planes in $\mathbb{P}^{N}$. Consider the incidence correspondence $\mathcal{U}:=\left\{\left(\mathbb{P}^{k}, p\right) \mid p \in \mathbb{P}^{k}\right\}$ with projection maps $\pi_{1}: \mathcal{U} \rightarrow \mathbb{G}(k, N)$ and $\pi_{2}: \mathcal{U} \rightarrow \mathbb{P}^{N}$. For every subvariety $W \subset \mathbb{G}(k, N)$, we denote $\mathcal{U}_{W}:=\pi_{1}^{-1}(W)$ and $X_{W}:=\pi_{2}\left(\mathcal{U}_{W}\right)$. Then $X_{W} \subset \mathbb{P}^{N}$ is a scroll if and only if $W$ is smooth and $\pi_{2}: \mathcal{U}_{W} \rightarrow X_{W}$ is an isomorphism. The following consequence of Terracini's lemma [14] will be useful. Let $\Sigma_{z} \subset X$ denote the entry locus of $z \in S X$, that is, the closure of the set $\left\{x \in X \mid \exists x^{\prime} \in X\right.$ with $\left.z \in\left\langle x, x^{\prime}\right\rangle\right\}$. We recall that $\operatorname{dim}\left(\Sigma_{z}\right)=\delta(X)$ for general $z \in S X$.

Lemma 3.1. Let $X \subset \mathbb{P}^{N}$ be a non-degenerate scroll over $W$ and let $z \in S X$ be a smooth point. If $\Sigma_{z} \cap F_{w} \neq \emptyset$ for every $w \in W$ then $S X=\mathbb{P}^{N}$.

Proof. Let $T_{z} S X \subset \mathbb{P}^{N}$ be the embedded tangent space to $S X$ at $z$. For every $w \in W$ there exists some $x \in \Sigma_{z} \cap F_{w}$, so $F_{w} \subset T_{x} X$. Then it follows from Terracini's lemma that $X=\cup_{w \in W} F_{w} \subset \cup_{x \in \Sigma_{z}} T_{x} X \subset T_{z} S X$. Since $X \subset \mathbb{P}^{N}$ is non-degenerate we deduce $T_{z} S X=\mathbb{P}^{N}$, and hence $S X=\mathbb{P}^{N}$.

The following lemma is well known. We include a short proof based on Lemma 3.1:

Lemma 3.2. Let $X \subset \mathbb{P}^{N}$ be a non-degenerate scroll over a curve. If $\delta(X)>0$ then $S X=\mathbb{P}^{N}$. 
Proof. Since $\operatorname{dim} \Sigma_{z}=\delta(X)>0$ for general $z \in S X$ and $\operatorname{dim} W=1$, we deduce that $\Sigma_{z} \cap F_{w} \neq \emptyset$ for every $w \in W$. Therefore $S X=\mathbb{P}^{N}$ by Lemma 3.1.

Let $X_{W} \subset \mathbb{P}^{N}$ be an $n$-dimensional scroll. It follows from the Barth-Larsen theorem that $\delta(X) \leq 2$. From now on, we will focus on the extremal case $\delta(X)=2$. On the one hand, if $W$ is a curve then $X_{W} \subset \mathbb{P}^{N}$ is the Segre embedding $\mathbb{P}^{1} \times \mathbb{P}^{n-1} \subset \mathbb{P}^{2 n-1}$ (see [8, pp. 307-308]). We prove this result in a more geometric and elementary way. The idea of the proof is essentially due to Fyodor Zak:

Proposition 3.1. The only $n$-dimensional scroll over a curve in $\mathbb{P}^{2 n-1}$ is the Segre embedding $\mathbb{P}^{1} \times \mathbb{P}^{n-1} \subset \mathbb{P}^{2 n-1}$.

Proof. For every $w \in W$, let $\sigma_{w}:=\left\{g \in \mathbb{G}(n-1,2 n-1) \mid \mathbb{P}_{g}^{n-1} \cap F_{w} \neq \emptyset\right\}$. Then $\sigma_{w}$ is a hyperplane section of $\mathbb{G}(n-1,2 n-1)$, embedded by Plücker, and $w \in \sigma_{w}$ is a singular point of multiplicity $n$. The intersection of $W$ and $\sigma_{w}$ is supported at $w$ since $\pi_{2}: \mathcal{U}_{W} \rightarrow X_{W}$ is injective. Moreover, $W$ and $\sigma_{w}$ meet transversally at $w$ since $d \pi_{2}$ is injective. So we deduce that $\operatorname{deg} X_{W}=\operatorname{deg} W=m_{w}\left(\sigma_{w}\right) \cdot m_{w}(W)=n$, where $m_{w}\left(\sigma_{w}\right)$ and $m_{w}(W)$ denote the multiplicity of $\sigma_{w}$ and $W$ at $w$, respectively (see [4, Corollary 12.4]). Therefore, for every $w \in W$ there exists a hyperplane section $\sigma_{w}$ in the Plücker embedding of $W$ such that the intersection product $\sigma_{w} \cdot W=n w$. This property characterizes the rational normal curve of degree $n$, so $X_{W} \subset \mathbb{P}^{2 n-1}$ is a non-degenerate (otherwise $F_{w} \cap F_{w^{\prime}} \neq \emptyset$ for every $w^{\prime} \in W$ ) rational normal scroll of degree $n$. Consequently, $X_{W}$ is the Segre embedding $\mathbb{P}^{1} \times \mathbb{P}^{n-1} \subset \mathbb{P}^{2 n-1}$.

Remark 3.1. The hypothesis of Proposition 3.1 can be weakened. Arguing with a general $w \in W$, the same proof works if $W \subset \mathbb{G}(n-1,2 n-1)$ is an integral curve and $\pi_{2}: \mathcal{U}_{W} \rightarrow X_{W}$ is an isomorphism (or even if $\pi_{2}: \mathcal{U}_{W} \rightarrow X_{W}$ has finitely many double points).

On the other hand, if $W$ is a surface there exists a complete classification of scrolls with $\delta(X)=2$ only for $n=3$ (see [7, Proposition $4 ; 12]$ ). We now prove that (an isomorphic projection of) the Segre embedding $\mathbb{P}^{2} \times \mathbb{P}^{n-2} \subset \mathbb{P}^{3 n-4}$ with $n \geq 4$ is the only scroll over a surface whose secant variety does not fill up the ambient space. The main idea of the proof is to show that $X \subset \mathbb{P}^{N}$ is swept out by a two-dimensional family of Segre embeddings $\mathbb{P}^{1} \times \mathbb{P}^{n-2}$. More precisely, we prove that any two fibres of the scroll, $F_{w}$ and $F_{w^{\prime}}$, determine a Segre embedding $\mathbb{P}^{1} \times \mathbb{P}^{n-2}$ in the linear span $\left\langle F_{w}, F_{w^{\prime}}\right\rangle=: \mathbb{P}_{w w^{\prime}}^{2 n-3}$ that they define.

Theorem 3.1. Let $X \subset \mathbb{P}^{N}$ be a non-degenerate scroll of dimension $n$ over a surface. If $\delta(X)=2$ and $S X \neq \mathbb{P}^{N}$ then $X$ is (an isomorphic projection of) the Segre embedding $\mathbb{P}^{2} \times \mathbb{P}^{n-2} \subset \mathbb{P}^{3 n-4}$.

Proof. We claim that $\operatorname{dim} S\left(F_{w}, X\right)=2 n-2$ for general $w \in W$. Since $\left\langle F_{w}, F_{w^{\prime}}\right\rangle=$ $S\left(F_{w}, F_{w^{\prime}}\right) \subsetneq S\left(F_{w}, X\right)$ for every $w^{\prime} \in W$, we get $\operatorname{dim} S\left(F_{w}, X\right) \geq 2 n-2$. Fix a general $z \in S X$. We deduce from Lemma 3.1 that $\Sigma_{z} \cap F_{w}=\emptyset$ for general $w \in W$, and hence $z \notin S\left(F_{w}, X\right)$. Therefore $S\left(F_{w}, X\right) \subsetneq S X$ proving the claim, as $\operatorname{dim} S X=$ $2 n-1$. For every $w \in W$, consider the subvariety $\mathcal{G}_{w}:=\left\{\left\langle F_{w}, F_{w^{\prime}}\right\rangle \mid w^{\prime} \in W\right\} \subset$ $\mathbb{G}(2 n-3, N)$. If $\operatorname{dim} \mathcal{G}_{w}=2$ for general $w \in W$ then $S\left(F_{w}, X\right) \subset \mathbb{P}^{N}$ is a $(2 n-2)$ dimensional subvariety swept out by a two-dimensional family of $(2 n-3)$-dimensional linear subspaces, so $S\left(F_{w}, X\right) \subsetneq \mathbb{P}^{N}$ itself is a linear subspace. This contradicts the 
non-degeneracy of $X \subset \mathbb{P}^{N}$. Thus $\operatorname{dim} \mathcal{G}_{w}=1$ for general (and hence every) $w \in W$. In particular, for every $w, w^{\prime} \in W$ there exists an integral curve $T_{w w^{\prime}} \subset W$ such that $\left\langle F_{w}, F_{w^{\prime}}\right\rangle=\left\langle F_{w}, F_{w^{\prime \prime}}\right\rangle$ for every $w^{\prime \prime} \in T_{w w^{\prime}}$. So $X_{T_{w w^{\prime}}}=\mathbb{P}^{1} \times \mathbb{P}^{n-2} \subset \mathbb{P}_{w w^{\prime}}^{2 n-3}$ by Remark 3.1. Consequently, for every $w \in W$ and every $x \in F_{w}$ there exists a one-dimensional family of lines each of them meeting $F_{w}$ at $x$ and giving a twodimensional cone $C_{x} \subset X$. Since $F_{w}$ and $C_{x}$ are contained in $T_{x} X=\mathbb{P}^{n}$ we deduce $\operatorname{deg}\left(C_{x}\right)=C_{x} \cdot F_{w}$. We claim that $C_{x} \cdot F_{w^{\prime}}=1$ for every $w^{\prime} \in W$, and hence $\operatorname{deg}\left(C_{x}\right)=C_{x} \cdot F_{w}=C_{x} \cdot F_{w^{\prime}}=1$. Let us prove the claim. If $C_{x} \cdot F_{w^{\prime}} \geq 2$ then $T_{x} X=\left\langle F_{w}, C_{x} \cap F_{w^{\prime}}\right\rangle \subset\left\langle F_{w}, F_{w^{\prime}}\right\rangle$. Therefore $T\left(F_{w}, X\right) \subset\left\langle F_{w}, F_{w^{\prime}}\right\rangle$, contradicting Theorem 1.1. Since $\operatorname{deg}\left(C_{x}\right)=C_{x} \cdot F_{w}=1$, we deduce that $C_{x}=\mathbb{P}^{2}$ for every $x \in F_{w}$ and that $C_{x}$ is a section of $\pi: X_{W} \rightarrow W$ (in particular, $W \cong \mathbb{P}^{2}$ ). Thus $X$ is a scroll over $\mathbb{P}^{2}$ and $\mathbb{P}^{2 n-2}$, respectively. So $X \subset \mathbb{P}^{N}$ is an isomorphic projection of the Segre embedding $\mathbb{P}^{2} \times \mathbb{P}^{n-2} \subset \mathbb{P}^{3 n-4}$.

\section{Acknowledgment}

The author is grateful to Fyodor Zak for helpful comments and encouragement. Research supported by the "Ramón y Cajal" contract RYC-2009-04999 and the project MTM2009-06964 of MICINN

\section{References}

[1] L. Ein, Varieties with small dual varieties II, Duke Math. J. 52 (1985), 895-907.

[2] L. Ein, Varieties with small dual varieties I, Invent. Math. 86 (1986), 63-74.

[3] T. Fujita, Projective threefolds with small secant varieties, Sci. Papers College Gen. Ed. Univ. Tokyo 32 (1982), 33-46.

[4] W. Fulton, Intersection theory, in 'Ergebnisse der Mathematik und ihrer Grenzgebiete. 3. Folge', 2nd ed., A Series of Modern Surveys in Mathematics, 2, Springer-Verlag, Berlin, 1998.

[5] W. Fulton and J. Hansen, A connectedness theorem for projective varieties, with applications to intersections and singularities of mappings, Ann. of Math. (2) 110 (1979), 159-166.

[6] P. Ionescu and F. Russo, Manifolds covered by lines, defective manifolds and a restricted Hartshorne conjecture, arXiv:0909.2763v2 [math.AG].

[7] P. Ionescu and M. Toma, Boundedness for some special families of embedded manifolds, in 'Classification of algebraic varieties (L'Aquila, 1992)', Contemp. Math., 162, Amer. Math. Soc., Providence, RI, 1994, 215-225.

[8] S. L. Kleiman, Plane forms and multiple-point formulas, in 'Algebraic threefolds (Varenna, 1981)', Lecture Notes in Math., 947, Springer, Berlin, 1982, 287-310.

[9] S. L. Kleiman, Tangency and duality, in Proceedings of the 1984 Vancouver Conference in Algebraic Geometry, CMS Conf. Proc., 6, Amer. Math. Soc., Providence, RI, 1986, 163-225.

[10] R. Lazarsfeld, Positivity in algebraic geometry I-II, in Ergebnisse der Mathematik und ihrer Grenzgebiete. 3. Folge, A Series of Modern Surveys in Mathematics, 48-49, Springer-Verlag, Berlin, 2004.

[11] S. Mukai, Biregular classification of Fano 3-folds and Fano manifolds of coindex 3, Proc. Natl. Acad. Sci. USA 86 (1989), 3000-3002.

[12] G. Ottaviani, On 3-folds in $\mathbf{P}^{5}$ which are scrolls, Ann. Scuola Norm. Sup. Pisa Cl. Sci. (4), 19 (1992), 451-471.

[13] C. Segre, Preliminari di una teoria delle varietà luoghi di spazi, Rend. Circ. Mat. Palermo, 30 (1910), 87-121.

[14] A. Terracini, Sulle $V_{k}$ per cui la varietà degli $S_{h}(h+1)$-seganti ha dimensione minore dell' ordinario, Rend. Circ. Mat. Palermo 31 (1911), 392-396.

[15] F. L. Zak, Projections of algebraic varieties, Mat. Sb. (N.S.), 116(158) (1981), 593-602, English translation in Mat. USSR Sb. 44 (1983), 535-544. 
[16] F. L. Zak, Tangents and secants of algebraic varieties, in 'Translations of mathematical monographs', 127, American Mathematical Society, Providence, RI, 1993.

Instituto de Ciencias Matemáticas (ICMAT), Consejo Superior de Investigaciones Científicas (CSIC), Campus de Cantoblanco, 28049 Madrid, Spain

E-mail address: jcsierra@icmat.es 
